\title{
Large-Scale Vortical Motion and Pressure Fluctuation in Noise-Controlled, Swirl-Stabilized Combustor*
}

\author{
Masayasu SHIMURA**, Mamoru TANAHASHI ${ }^{* *}$, Gyung-Min CHOI $^{* * *}$ and \\ Toshio MIYAUCHI** \\ ** Department of Mechanical and Aerospace Engineering, Tokyo Institute of Technology \\ 2-12-1 Ookayama, Meguro-ku, Tokyo 152-8550, Japan \\ E-mail: mshimura@navier.mes.titech.ac.jp \\ *** School of Mechanical Engineering, College of Engineering, Pusan National University \\ Busan 609-735, Republic of Korea
}

\begin{abstract}
To clarify the mechanisms of combustion oscillation, combustion noise and their suppression by secondary fuel injection, simultaneous measurements of stereoscopic particle image velocimetry (SPIV) and pressure fluctuation in combustion chamber were conducted on several planes of a swirl-stabilized turbulent premixed flame for no control case and noise-controlled case by continuous secondary fuel injection. Velocities measured by SPIV were averaged in 8 phases of the pressure fluctuation and streamlines were obtained from the phase-averaged velocities. For no control case, large-scale vortical structures are generated in regions around the axial centerline of the combustor and outer edge of the swirl nozzle in the phase of low pressure. With increasing pressure, they move near the contour surface of mean progress variable $\bar{c}=0.5$ which have been obtained in the previous study. These large-scale vortical motions induce fluctuation of flame front and enhance entropy term in the acoustic sound source. The secondary fuel injection suppresses the velocity fluctuation in the inner recirculation zone, resulting in reduction of combustion noise. These results show that control of the large-scale vortical motion is important for reduction of combustion noise.
\end{abstract}

Key words : Turbulent Combustion, Combustion Oscillation, Combustion Noise, Combustion Control, Secondary Fuel Injection, SPIV

\section{Introduction}

Lean premixed combustion which is a candidate for high-efficiency and low-emissions gas turbines is prone to combustion oscillations. Rayleigh ${ }^{(1)}$ showed that combustion oscillations are mainly caused by the feedback interaction between natural acoustic modes of the combustor and oscillation of heat release rate. Mathematical models of the combustion instabilities have been reviewed by McManus et al. ${ }^{(2)}$. To obtain important factors for control of combustion oscillations, quantitative measurements of the flame response to acoustic perturbations have been conducted by Poinsot et al. ${ }^{(3)}$ and Harper et al. ${ }^{(4)}$. Flame-acoustic interactions in unstable combustors have been investigated by Samaniego et al. ${ }^{(5)}$, Broda et al. ${ }^{(6)}$ and Balachandran et al. ${ }^{(7)}$ using phase-locked measurements. In general, these results show the significant influence of the combustor geometry or acoustic properties of the combustion system on the unstable phenomena.

Sound generation mechanisms in turbulent reactive flows, which are closely related to combustion instabilities, have been investigated by direct numerical simulations ${ }^{(8)-(11)}$. In general, acoustic sound source can be decomposed into a Reynolds stress term, an entropy term and a viscous term. It is well-known that the entropy term is a main sound source in combustion field and is dominated by fluctuation of heat release rate. In addition, the entropy 
term from the turbulent energy dissipation rate is also important ${ }^{(9),(10)}$. The distribution of entropy term is determined by the energy dissipation rate in the area where the heat release rate is very small. Fine scale eddies are closely related to the entropy term, because turbulent energy dissipation rate is very high around fine scale eddies ${ }^{(12),(13)}$. The fine scale eddies are also closely related with the Reynolds stress term ${ }^{(10)}$. Therefore, fine scale eddies of turbulence are important sound source in three-dimensional turbulent flows. These facts suggest that the key point to develop a control scheme of combustion noise is how to suppress the fluctuations of heat release rate and how to control the fine scale eddies of turbulence which have a close relation with turbulent energy dissipation rate.

Combustion control by secondary fuel injection has been studied by many researchers ${ }^{(14)}$. Hong et al. ${ }^{(15)}$ have conducted experiments to evaluate the control law under the wide-range operation of a generic combustor using secondary fuel injection. Lee et al. ${ }^{(16)}$ have investigated effects of secondary fuel injection location on the effectiveness of active combustion control in a laboratory-scale dump combustor at atmospheric pressure. Tachibana et al. ${ }^{(17)}$ have also studied the effect of secondary fuel injection localtion on combustion control. In the previous study ${ }^{(18)}$, we have revealed that, due to the modification of local flame structure by secondary fuel injection, combustion noise and $\mathrm{NO}_{\mathrm{X}}$ emission can be reduced simultaneously. Furthermore, Tanahashi et al. ${ }^{(19)}$ have shown that combustion noise is further suppressed by intermittent secondary fuel injection and controlling injection frequency. It is worth to note that the optimum frequency is much lower than the natural acoustic mode of the combustor in Tanahashi et al. ${ }^{(19)}$. Uhm et al. ${ }^{(20)}$ have also reported that low-frequency modulation periodically alters the phase relation between pressure and heat release.

However, to achieve more effective control of oscillating combustion, it is necessary to get detailed information about flame structure and turbulent structure in stable and unstable conditions of combustor. 3D flame structures of turbulent premixed flames have been investigated by $\mathrm{OH}$ planar laser induced fluorescence (PLIF) in our previous study ${ }^{(21)}$. It was revealed that the secondary fuel injection reduces fluctuation of flame front in flame zone and that of high temperature gas in recirculation zone. Dawson et al. ${ }^{(22)}$ investigated the destabilizing effect of combustion instability on the shape and size of recirculation zones by measuring velocity field with a laser Doppler velocimetry (LDV) and pressure. Weigand et al. ${ }^{(23)}$ conducted LDV, PLIF of $\mathrm{OH}$ and $\mathrm{CH}$, and laser Raman scattering in swirling methane-air lean diffusion flames to understand the physical and chemical processes leading to the different behavior of the flames. Armitage et al. ${ }^{(24)}$ investigated acoustically forced lean premixed turbulent bluff-body stabilized flames by using CFD of turbulent combustion and comparing that with their experimental work $^{(7)}$.

The objectives of this study are to investigate the relation between the large-scale vortical motion and pressure fluctuation in a noise-controlled swirl-stabilized combustor, and to reveal the mechanisms of combustion oscillation, combustion noise and their suppression by secondary fuel injection. In this study, simultaneous measurements of SPIV and pressure fluctuation in combustion chamber were conducted on several planes of the combustor, and large vortical structures were extracted by the phase-averaged velocity maps.

\section{Experimental Method}

\subsection{Experimental Apparatus}

In this study, a swirl-stabilized combustor which has been investigated in our previous works $^{(18),(19),(21)}$ is used. Figure 1 shows the schematic of the swirl-stabilized combustor and details of secondary fuel injection nozzle. This combustion rig consists of a contraction section, a swirl nozzle section and a combustion chamber. The inner diameter of a plenum is reduced from $120 \mathrm{~mm}$ to $40 \mathrm{~mm}$ in the contraction section. The swirl nozzle of $40 \mathrm{~mm}$ inner diameter is mounted on contraction section. The inner cross-section of combustion chamber is $120 \mathrm{~mm} \times 120 \mathrm{~mm}$, and the outlet of combustion chamber is contracted to $60 \mathrm{~mm} \times 60$ $\mathrm{mm}$. The total length of the chamber is $590 \mathrm{~mm}$. On each side of combustion chamber, 


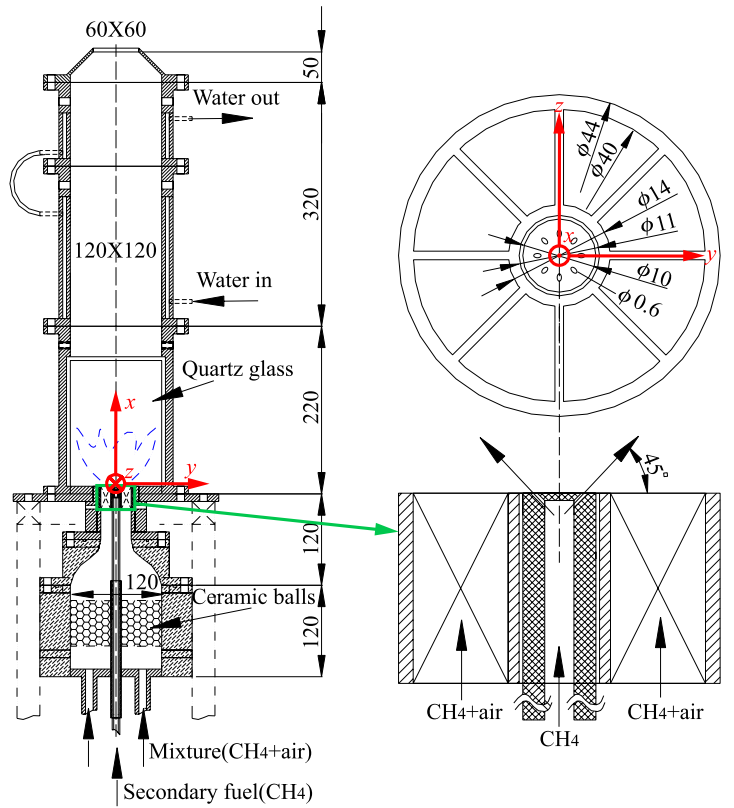

Fig. 1 Schematics of swirl-stabilized combustor and details of swirl vanes and secondary fuel injection holes.

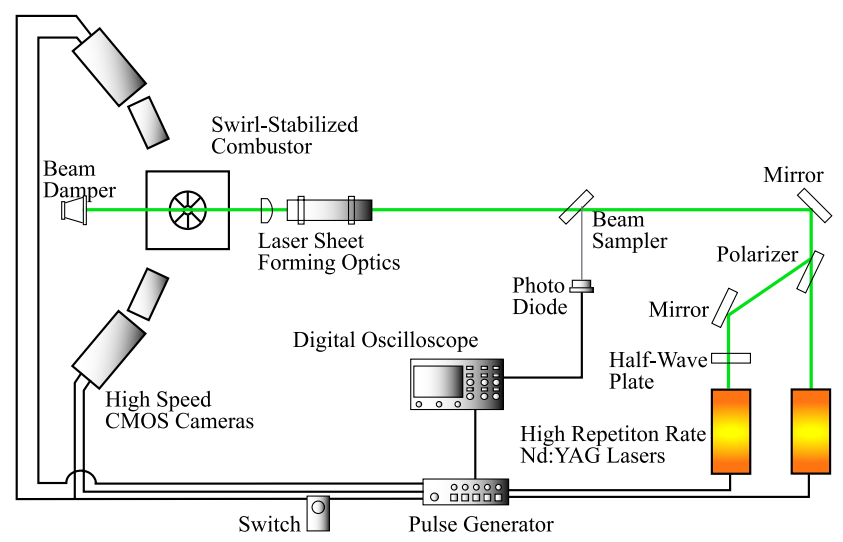

Fig. 2 Schematic diagram of SPIV system.

a silica glass plate of $120 \mathrm{~mm} \times 170 \mathrm{~mm}$ and $5 \mathrm{~mm}$ thickness is installed to allow optical access. Combustion chamber upper than $220 \mathrm{~mm}$ is cooled by water. The swirl nozzle has 8 swirl vanes of $14 \mathrm{~mm}$ inner diameter and $40 \mathrm{~mm}$ outer diameter, inclined $45^{\circ}$ from the nozzle axis. Methane-air premixed gas, which is not heated, passes through the swirl vanes and the flame is stabilized at the swirl vanes. The secondary fuel nozzle is mounted at the hub of the swirler and has 8 injection holes (ID $=0.6 \mathrm{~mm}$ ) inclined at $45^{\circ}$ to the mainstream direction. The secondary fuel injection can be modulated by a solenoid operated valve (MAC Valves, 34BABA-GEME-1BA). The tap of injection holes is connected to the valve by a straight pipe and a flexible pipe. The distance between the tap and the valve is about $750 \mathrm{~mm}$. The response time of the fluid at the nozzle exit depends on flow rate. In this study, the valve is driven by direct current for continuous injection and is always open for the direct current.

\subsection{Experimental Set-up of Stereoscopic Particle Image Velocimetry and Pressure Fluc- tuation Measurement}

Figure 2 shows a schematic diagram of SPIV system. The system consists of two highrepetition-rate Nd:YAG lasers (Lee Laser, LDP-100MQG, $50 \mathrm{~W}$ at $10 \mathrm{kHz}$ ), several optics, two high-speed CMOS cameras (Vision Research, Phantom V7.1, $800 \times 600$ pixels) and a pulse/delay generator (Labsmith, LC880). Two laser beams become double-pulsed through 


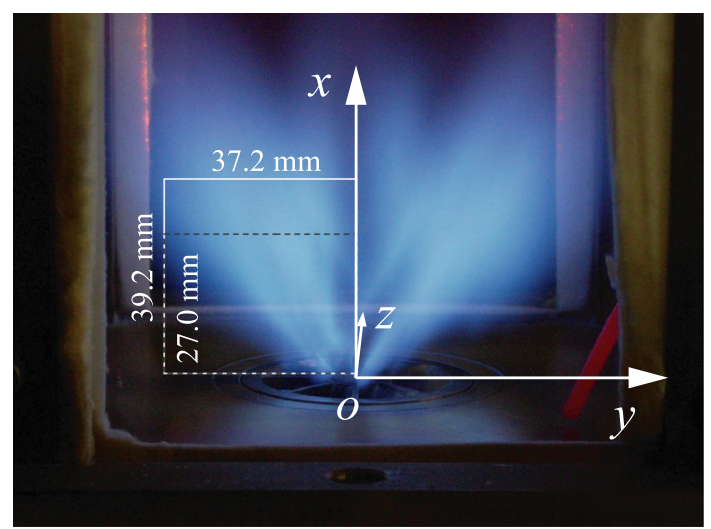

Fig. 3 Long-time exposure photo of methane-air turbulent premixed flame $\left(Q_{\mathrm{m}}=300\right.$ $\mathrm{L} / \mathrm{min}$ ) controlled by continuous secondary fuel injection $\left(Q_{\mathrm{sf}}=3 \mathrm{~L} / \mathrm{min}\right)$. Gray dotted box and white box are measurement regions including centerline of the combustor for no control case and continuous injection case respectively.

the laser beam combining optics which include a half-wave plate (CVI Laser) and a polarizer (CVI Laser). Time interval $(\Delta t)$ of double-pulsed beams is confirmed by a photo diode and a digital oscilloscope. The laser beams were expanded by the laser sheet forming optics, and were scattered by $2 \mu \mathrm{m} \mathrm{SiO} 2$ particles mixed in the methane-air premixed gas. Scattered light was detected by the CMOS cameras with telephotographic lens (Nikon, Micro-Nikkor 200 $\mathrm{mm} / \mathrm{f} 4)$. The CMOS cameras are located with $19^{\circ}$ inclined forward with respect to normal to the measurement plane to capture forward scattered light. In order to obtain particle images in good focus over the whole measurement region, the Scheimpflug condition ${ }^{(25)}$ was used.

Pressure transducer (JTEKT, PD104) was used to measure pressure fluctuation in the combustor. A water-cooling connection tube was used to attach the pressure transducer to the combustion chamber at $500 \mathrm{~mm}$ downstream from the exit of swirl nozzle. Pressure signal, a start signal of measurement and strobe signal of CMOS camera were simultaneously recorded by PC with A/D converter (National Instruments, PCI-6115). Sampling rate was set to 200 $\mathrm{kHz}$ to detect strobe signal which is shorter than $10 \mu \mathrm{sec}$.

\subsection{Calculation of Velocity}

For distortion compensation, 2D calibration ${ }^{(26)}$ was applied to particle images recorded by each camera. Instantaneous velocity map was calculated by 2 -step hierarchical method ${ }^{(27)}$ and a window-offset method ${ }^{(28)}$. Overlap of interrogation regions was set to $80 \%$. Spatial cutoff filter which has been developed by our previous study ${ }^{(29)}$ was used to eliminate highwave number noises introduced by the overlap of the interrogation windows. The accuracy of the PIV measurement with the elimination scheme of spurious vectors and noise has been ensured by a PIV simulation based on DNS of particle-laden homogeneous isotropic turbulence in the previous study ${ }^{(30)}$. From two-dimensional velocity fields obtained by each camera, three-component velocity vectors on a two-dimensional plane were calculated by using a geometrical relation $^{(26),(31)}$.

\subsection{Experimental Conditions}

Measurements were conducted without secondary fuel injection, which is referred as no control case, and with continuous secondary fuel injection, which is called continuous injection case hereafter. The flow rate of the main methane-air mixture $\left(Q_{\mathrm{m}}\right)$ was selected to 300 $\mathrm{L} / \mathrm{min}$ for both cases. Equivalence ratio $(\phi)$ of main mixture was set to 0.790 for no control case. As for the case of continuous injection, equivalence ratio of main mixture was 0.717 and flow rate of the secondary fuel injection $\left(Q_{\mathrm{sf}}\right)$ was set to $1 \%$ of $Q_{\mathrm{m}}$ (total $\phi=0.819$ ). Figure 3 shows measurement regions of SPIV on a long-time exposure photo of methane-air premixed flame with continuous secondary fuel injection. The $x, y$ and $z$ axes of coordinates in mea- 
(a)

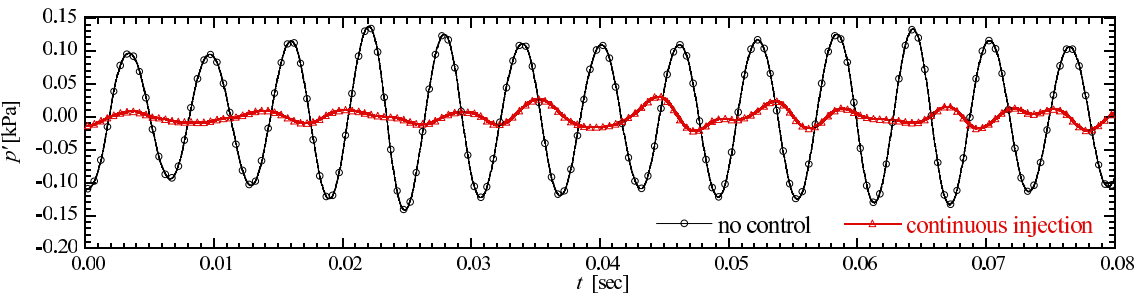

(b)

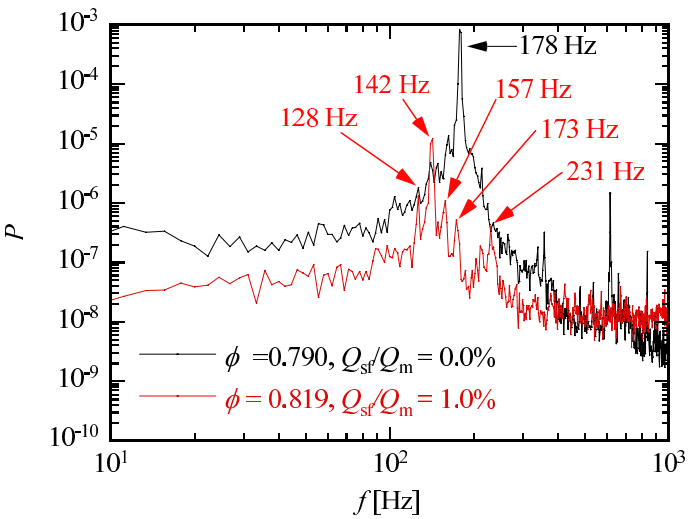

Fig. 4 Characteristics of pressure fluctuation in the combustion chamber for no control and continuous secondary fuel injection cases. (a) An example of time series data. (b) Power spectra.

surement region are set to main stream direction of the combustor, laser travel direction and normal direction to the other axes, respectively, and origin of the coordinate system is set to the center of swirler exit. Gray dotted box in Fig. 3 corresponds to a whole measurement region including centerline of the combustor for no control case. The size of the region is $27.0 \times 37.2$ $\mathrm{mm}^{2}$. The measurement region is located $2 \mathrm{~mm}$ above the swirler exit, and central position of the region is $(15.5 \mathrm{~mm},-18.6 \mathrm{~mm}, 0 \mathrm{~mm})$. The measurement region was divided into four rectangles which overlap $2.5 \mathrm{~mm}$ each other, and SPIV measurements were conducted on each area in order to improve spatial resolution of SPIV. White box in Fig. 3 corresponds to a measurement region for continuous injection case. The size of this region is $39.2 \times 37.2 \mathrm{~mm}^{2}$ and central position of the region is $(21.6 \mathrm{~mm},-18.6 \mathrm{~mm}, 0 \mathrm{~mm})$. The measurement region was divided into six rectangles on which SPIV measurements were conducted. Flame zone for continuous injection case was pushed downstream by secondary fuel injection ${ }^{(21)}$. These measurement regions cover recirculation zone and flame zone, and were moved from the plane including the centerline of the combustor to the edge of the combustor with the distance of 4 $\mathrm{mm}$. Total number of the measurement planes is 5 in one side of the combustor for no control case $(z=0 \sim-16 \mathrm{~mm})$. As for continuous injection case, SPIV was conducted on the planes of $z=0 \mathrm{~mm}$ and $-16 \mathrm{~mm}$. The high-repetition-rate Nd:YAG lasers were operated at 4 $\mathrm{kHz}$ with $3 \mathrm{~mJ} / \mathrm{pulse}$, and camera resolution was set to the maximum $800 \times 600$ pixels. The high-repetition-rate measurements reduce background noise caused by chemiluminescence. Spatial resolution of SPIV was $1.23 \mathrm{~mm}$, and $\Delta t$ was altered in accordance with turbulence and turbulent combustion characteristics of measurement region $(15 \sim 30 \mu \mathrm{sec})$.

\section{Pressure Fluctuation in the Swirl-Stabilized Combustor}

The characteristics of pressure fluctuation in the combustor are shown in Fig. 4. Figure 4 (a) shows an example of time-series pressure fluctuation for no control and continuous injection cases. Pressure signal is filtered by low-pass filter of $500 \mathrm{~Hz}$. The pressure fluctuation is suppressed by continuous secondary fuel injection drastically. Figure 4 (b) shows power spectra obtained from raw pressure data. Dominant peak frequency for no control case is $178 \mathrm{~Hz}$. As for continuous injection case, dominant peak frequency changes to $142 \mathrm{~Hz}$, and energy at 


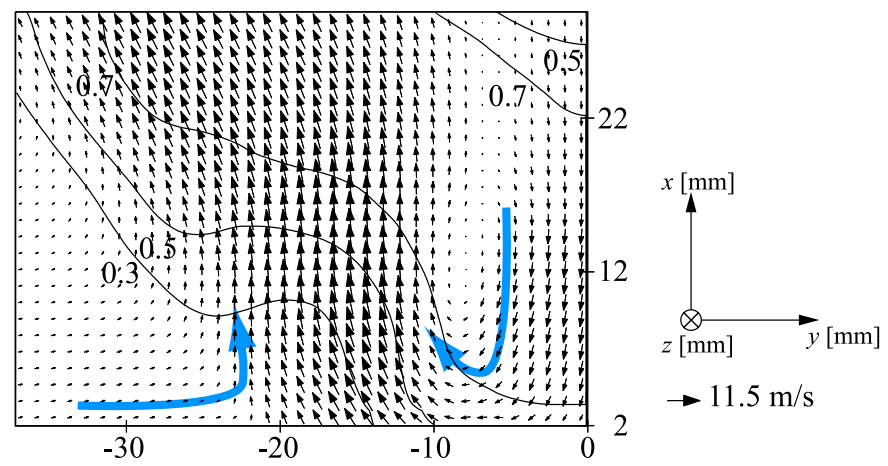

Fig. 5 Two-dimensional mean velocity map on $z=0 \mathrm{~mm}$ for no control case. Solid lines represents iso-lines of mean progress variable $\bar{c}=0.3,0.5$ and $0.7^{(21)}$.

(a)
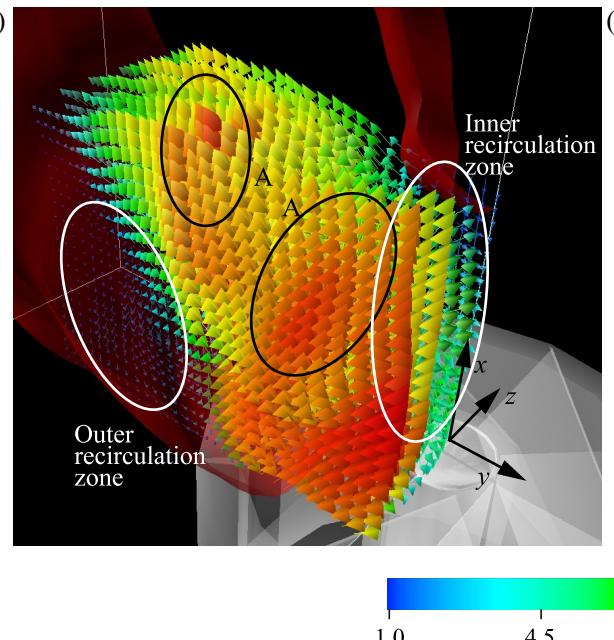

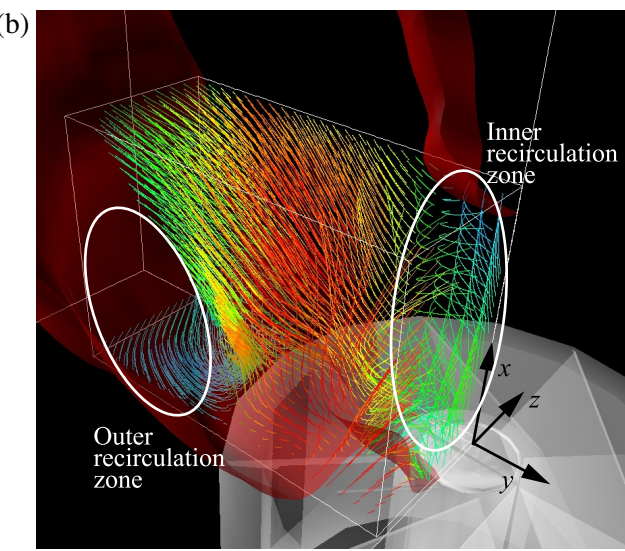

$11.5[\mathrm{~m} / \mathrm{s}]$

Fig. 6 Three-dimensional mean velocity field (a) and streamlines (b) obtained from the mean velocity for no control case. Magnitude of velocity is represented by color, and size of vectors is illustrated to be proportional to the magnitude of velocity. Red surfaces correspond to $\bar{c}=0.5$ obtained in the previous study ${ }^{(21)}$.

the peak frequency is lower than that for no control case. However, new additional peaks are induced at 128,157, 173 and $231 \mathrm{~Hz}$. These results indicate that continuous secondary fuel injection does not sufficiently suppress combustion oscillation and that alternative feedback interaction is induced by secondary fuel injection. This alternative feedback interaction can be eradicated by injecting secondary fuel intermittently and controlling injection frequency ${ }^{(21)}$. In this study, velocities obtained by SPIV were averaged in 8 phases of the pressure oscillation. Here, the phase of SPIV data was estimated by finding local extrema of filtered pressure data $(\leq 500 \mathrm{~Hz})$ and the velocity data is separated into 8 phase bins (denoted by $\Delta$ ).

\section{Large-Scale Vortical Motions in the Swirl-Stabilized Combustor}

Figure 5 shows two-dimensional mean velocity map on $z=0 \mathrm{~mm}$ for no control case. Mean velocity was obtained from 2,400 instantaneous data. Black solid lines represents isolines of mean progress variable $\bar{c}=0.3,0.5$ and 0.7 obtained in the previous OH PLIF measurement ${ }^{(21)}$. The regions between $\bar{c}=0.3$ and 0.7 can be considered to be active flame zones (flame brush). Velocity of fluid increases through iso-lines of mean progress variable, i.e., flame brush. Recirculation flows are formed near the axial centerline of the combustor and near the wall of combustion chamber as shown by large arrows. Figure 6 shows threedimensional mean velocity field and streamlines obtained from the mean velocity for no control case. Red surfaces in Fig. 6 represent contour surfaces of mean progress variable $\bar{c}=0.5$ obtained in the previous study ${ }^{(21)}$. The largest red vector represents maximum velocity of 11.5 $\mathrm{m} / \mathrm{s}$. Although velocity of fluid increases through flame surface, there are low velocity regions 

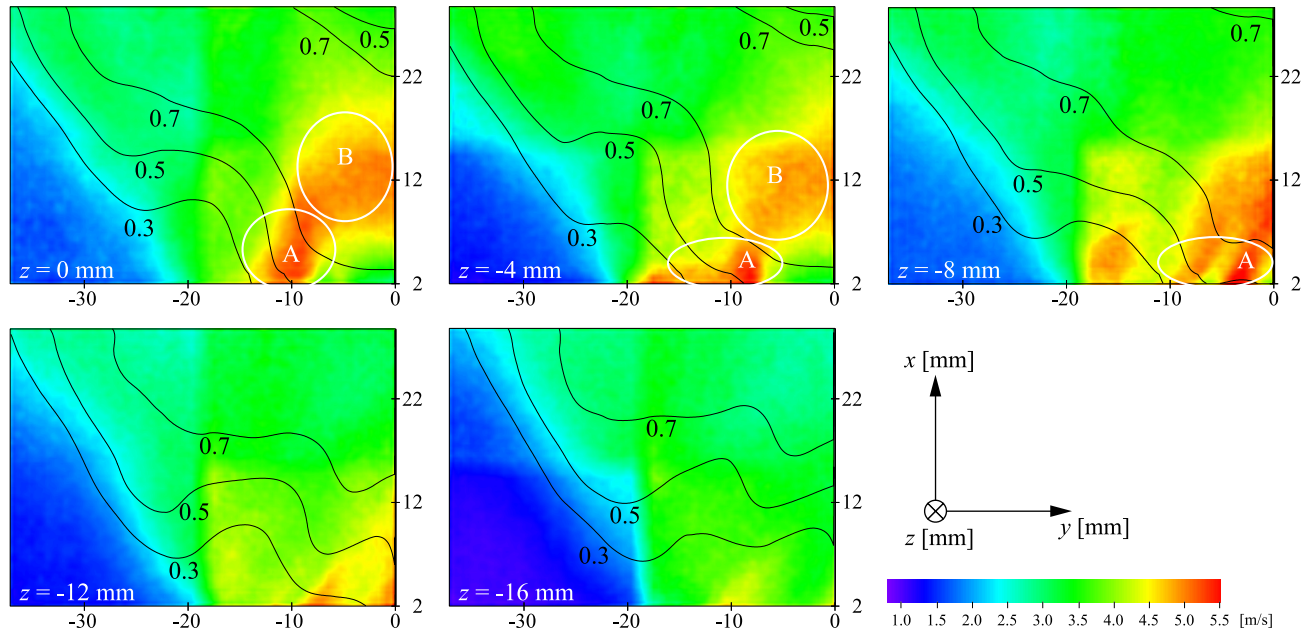

Fig. 7 Turbulent intensity on each measurement plane for no control case. Solid lines in each plane represents iso-lines of mean progress variable $\bar{c}=0.3,0.5$ and $0.7^{(21)}$.

which indicates existence of shear layers due to the swirl nozzle indicated by region A in Fig. 6 . These results also show that inner and outer recirculation zones, which are clearly shown in Fig. 5, have strongly three-dimensionality and show large radial velocity component.

Figure 7 shows rms of velocity fluctuation ( $u_{\mathrm{rms}}^{\prime}$ : turbulent intensity) on each measurement plane for no control case. Black solid lines in Fig. 7 represents iso-lines of mean progress variable $\bar{c}=0.3,0.5$ and $0.7^{(21)}$. Turbulent intensity near exit of the swirl nozzle shows high value on the plane of $z=0,-4$ and $-8 \mathrm{~mm}$ as is denoted by region A. These high turbulent intensity regions overlap with flame brush near the exit of swirl nozzle. Furthermore, turbulent intensity shows high value in the inner recirculation zone on the plane $z=0$ and $-4 \mathrm{~mm}$ (see region B), which denotes that recirculating high temperature gas fluctuates considerably in space and time. These turbulent fluctuations induce fluctuation of flame front and resultant pressure fluctuation in the combustor.

In this experimental condition, flow structures oscillates in specific resonance modes of the combustor. Figure 8 shows variations of streamlines which are obtained from the phaseaveraged velocity maps. Red surface indicates the contour surface of mean progress variable $\bar{c}=0.5$ reported in the previous study ${ }^{(21)}$. Since the mean progress variable is not phaseaveraged one, same contour surface is shown in all phases. Each of phase-averaged velocity was obtained from about 300 instantaneous data. Color of streamlines are illustrated with the same way as Fig. 6. The diagram at the bottom of Fig. 8 defines the phase of pressure oscillation in the combustor. Region A in Fig. 8 represents the inlet of unburnt mixture. $\Delta=0 \sim \pi / 4$ corresponds to the phase in which pressure is relatively high and decreasing. In this phase, velocity in burnt region is high and inlet velocity is relatively low. The size of inner recirculation zone near the hub is relatively small (region B). With the decrease of pressure in the combustion chamber $(\Delta=\pi / 4 \sim 3 \pi / 4)$, the inlet velocity increases. Inner and outer recirculation zones in Figs. 5 and 6 correspond to the regions B and C in Fig. 8, respectively. Inner and outer large-scale vortical structures are formed in the phase of $\Delta=\pi / 2 \sim 3 \pi / 4$. $\Delta=3 \pi / 4 \sim 5 \pi / 4$ corresponds to the phase in which pressure is relatively low and gets local minimum. In this phase, the large-scale vortical structures develop and move downstream with rising of pressure in the combustion chamber. In the phase of $\Delta=5 \pi / 4 \sim 2 \pi$ in Fig. 8 , the inlet velocity decreases with the increase of pressure in the combustion chamber. The outer large-scale vortical structure moves and fades away downstream. As for inner recirculating flow, magnitude of velocity decreases, and the inner large-scale vortical structure diminishes gradually.

Flame structure of this experimental condition is classified into corrugated flamelets regime of turbulent combustion diagram proposed by Peters ${ }^{(32)}$. In this regime, local flame 

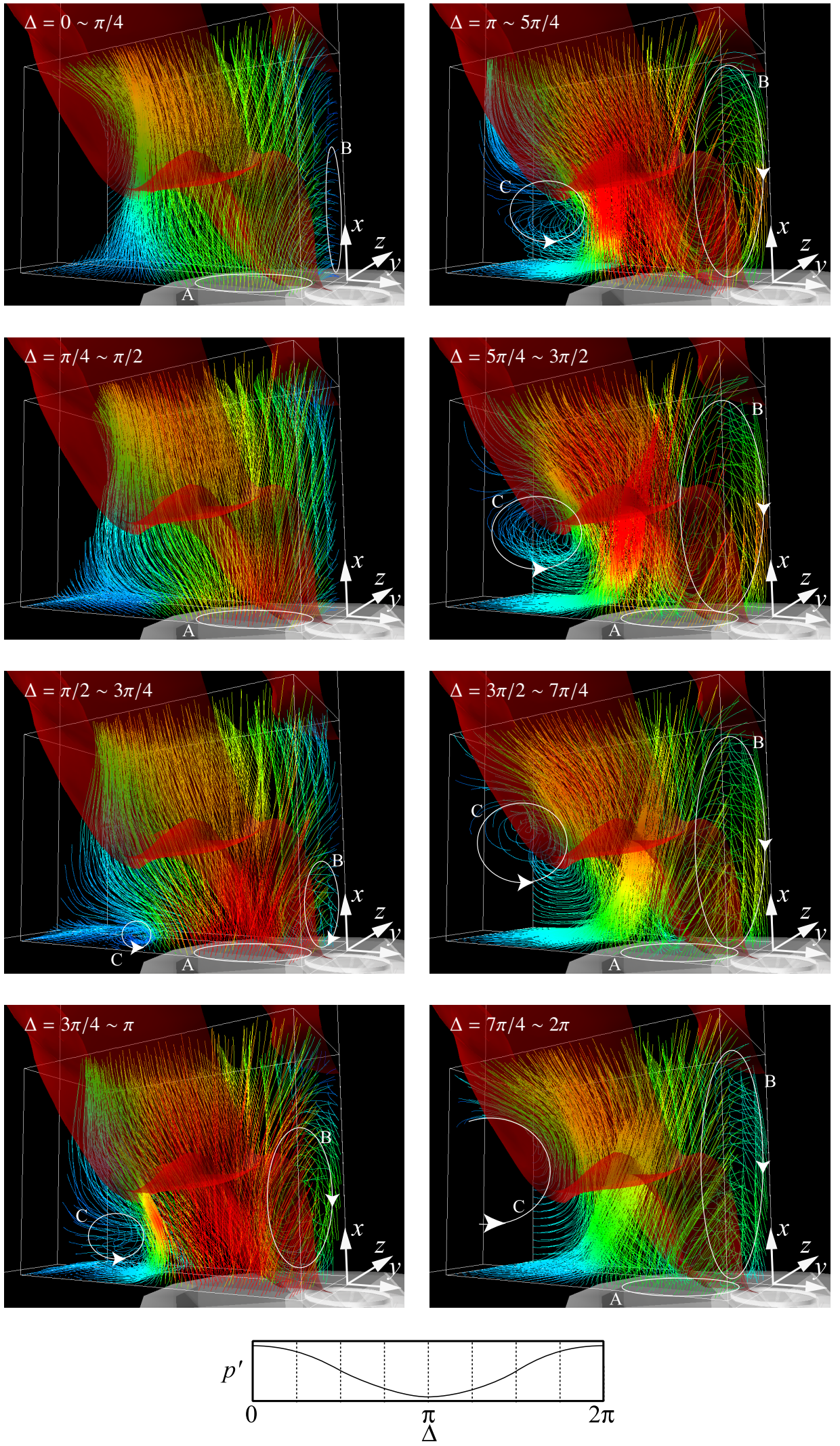

Fig. 8 Distributions of streamlines obtained from the phase-averaged velocity with the contour surface of mean progress variable $\bar{c}=0.5$ obtained in the previous study ${ }^{(21)}$. Diagram at the bottom defines the phase of pressure oscillation in the combustor. 

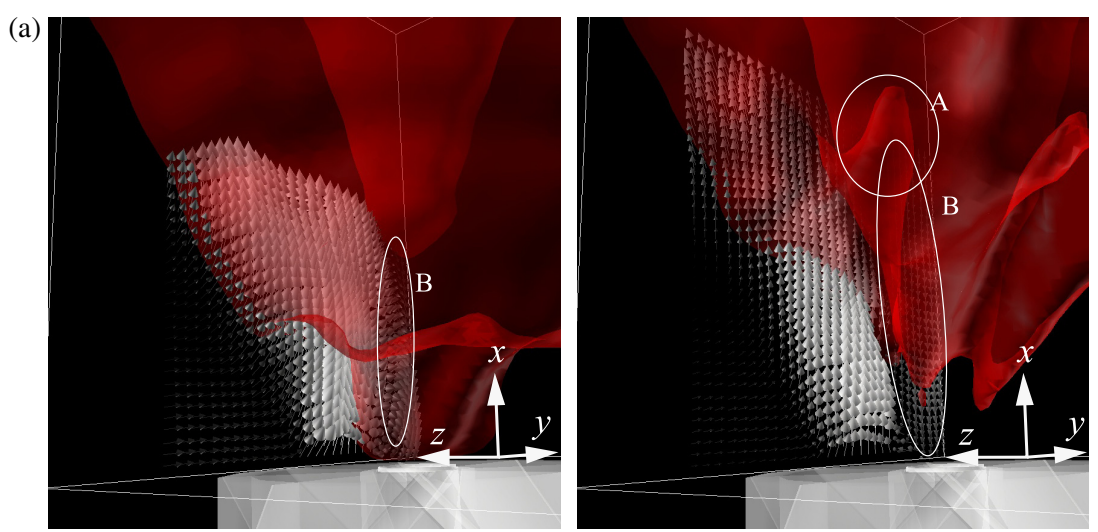

(b)
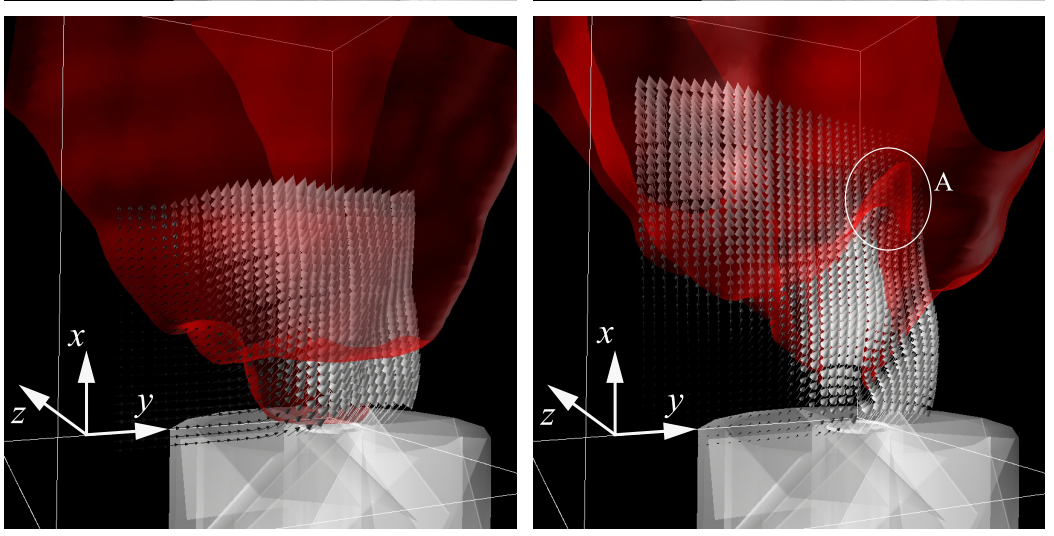

Fig. 9 Mean velocity map on the measurement planes of $z=0$ (a) and $-16 \mathrm{~mm}$ (b) for no control case (left) and continuous injection case (right). Red surfaces correspond to $\bar{c}=0.5$ which have been obtained in the previous study ${ }^{(21)}$.

elements can be considered to be laminar flamelets, and local heat release rate $(q)$ is approximated to be that of laminar flame. Therefore, instantaneous distribution of heat release rate is determined by the distribution of flame surface. Since the vortical structures are moving near the contour surface of $\bar{c}=0.5$, they induce the fluctuation of flame front, which causes fluctuation of heat release rate. It should be noted that, after linearization of continuity and energy conservation equations, entropy term $\left(T_{\mathrm{E}}\right)$ in acoustic sound source can be approximated by $T_{\mathrm{E}} \propto \partial^{2} q / \partial x_{i} \partial x_{j}^{(8)}$. Therefore, the vortical structures enhance entropy term of the acoustic sound source. The results obtained in this section suggest that suppression of the large-scale vortical motion formed in the recirculation zones is important for the reduction of flame front fluctuation and combustion noise. In addition, during one period of the pressure oscillation, inner large-scale vortical structure remains in near the exit of swirl nozzle with changing its size, and turbulent intensity in the inner recirculation zone is higher than that in the outer recirculation zone. Therefore, injection of secondary fuel to inner recirculation zone is more effective to suppress combustion oscillation. This consideration is supported by the previous studies ${ }^{(16),(17)}$. Lee et al. ${ }^{(16)}$ have shown that secondary fuel injection from dump plane to downstream results in insufficient suppression of pressure fluctuation compared with the injection to the inner recirculation zone. Tachibana et al. ${ }^{(17)}$ have reported that secondary fuel injection perpendicular to the main stream direction is less effective than the injection inclined at under $45^{\circ}$ from the axis of combustor.

\section{Suppression of Large-Scale Vortical Motion by Secondary Fuel Injection}

Figure 9 shows mean velocity maps on the measurement planes of $z=0$ and $-16 \mathrm{~mm}$ with contour surface of $\bar{c}=0.5$ for no control and continuous injection cases. Region of $\bar{c}=0.5$ spreads outwards and is pushed up by the jets of the secondary fuel for continuous injection case. No flame regions intrude into the downstream and concave structures are 
(a)

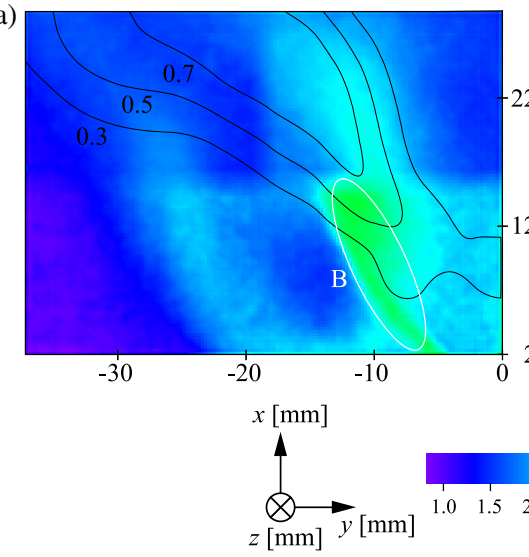

(b)

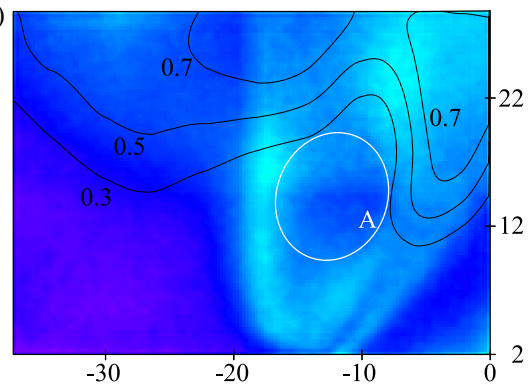

Fig. 10 Distributions of turbulent intensity for continuous injection case on the plane of $z=0$ (a) and $-16 \mathrm{~mm}$ (b). Solid lines in each plane represents iso-lines of $\bar{c}=0.3,0.5$ and $0.7^{(21)}$.

formed $^{(21)}$ as is shown by region A. Compared with no control case, the region of recirculation zone for continuous injection case is expanded as shown by region $\mathrm{B}$, and magnitude of fluid velocity on the plane of $z=0$ is decreased in near swirler exit. On the plane of $z=-16 \mathrm{~mm}$, fluid with high velocity blows into flame and establishes the concave structures. On the other hand, low velocity region can be observed between the concave structures.

Figure 10 shows distribution of turbulent intensity on the measurement planes of $z=0$ and $-16 \mathrm{~mm}$ for continuous injection case. Solid lines in each plane represents iso-lines of mean progress variable $\bar{c}=0.3,0.5$ and 0.7 . Figure 10 (a) shows that continuous secondary fuel injection suppresses the turbulent intensity around the inner recirculation zone and near the exit of swirl nozzle. In Fig. 10 (b), turbulent intensity is relatively low in the upstream of concave structure (see region A). By adding secondary fuel continuously, turbulent intensity was reduced to approximately half of no control case over all measurement regions. In the previous study ${ }^{(18)}$, it has been revealed that noise is reduced about $15 \mathrm{~dB}$ by continuous secondary fuel injection compared with that generated in the similar equivalence ratio without secondary fuel injection $(\phi=0.790)$. The secondary fuel injection suppresses flame front fluctuation and resultant fluctuation of heat release rate by reducing the velocity fluctuations. This suppression contributes to reduction of the entropy term in the acoustic sound sources. Furthermore, suppression of inlet velocity fluctuation corresponds to reduction of fine scale eddies and decreases Reynolds stress term induced by turbulent motion and the entropy term due to energy dissipation rate.

Here, active combustion control effective for suppression of combustion oscillation is discussed. According to Rayleigh ${ }^{(1)}$, suppression of combustion oscillation can be accomplished by introducing an energy source out of phase with combustion oscillation. However, combustion control with phase-shifting often lacks robustness, since practical combustors have a lot of instability modes. On the other hand, in the previous study ${ }^{(19)}$, the intermittent secondary fuel injection can suppress combustion noise and pressure fluctuation to the lower level than the continuous injection. In Fig. 10 (a), turbulent intensity in a region near the secondary fuel injection hole is higher than the surroundings as shown by region $\mathrm{B}$. This fluctuation works to induce new peaks of pressure fluctuation in Fig. 4 (b). However, intermittent secondary fuel injection can prevent the self-excited fluctuation by secondary fuel ${ }^{(21)}$. Note that the frequency of the intermittent injection is lower than the dominant instability mode, and that the suppression has been achieved in the wide range of injection frequency except for half of the dominant instability frequency. Therefore, selection of an appropriate frequency so as to keep a certain physical quantity minimal value can be one of robust approaches to suppress combustion noise and oscillation. 


\section{Conclusions}

To investigate the mechanisms of combustion oscillation, combustion noise and their suppression by secondary fuel injection, simultaneous measurements of SPIV and pressure fluctuation were conducted on multiple planes of methane-air turbulent premixed flames in the swirl-stabilized combustor for no control and continuous secondary fuel injection cases. Velocities measured by SPIV were averaged in 8 phases of the pressure fluctuation and streamlines were obtained from the phase-averaged velocity maps.

For no control case, large-scale vortical structures are generated in regions around the axial centerline of the combustor and outer edge of the swirl nozzle in the pressure decreasing phase. The vortical structures develop with moving downstream in the phase of the lowest pressure. They move near the contour surface of mean progress variable $\bar{c}=0.5$ in the pressure increasing phase. In the phase of high pressure, the outer large-scale vortical structure fades away downstream and the inner one diminishes gradually. These vortical motion induce flame front fluctuation, i.e., fluctuation of heat release rate, and enhance entropy terms in the acoustic sound source. Therefore, suppression of the large-scale vortical motion is important for reduction of combustion noise.

The secondary fuel injection suppresses the velocity fluctuation around the inner recirculation zone and that near the exit of swirl nozzle. These suppressions cause reduction of flame front fluctuation and fluctuation of heat release rate. Therefore, secondary fuel injection contributes to reduction of the entropy term in the acoustic sound sources. Furthermore, suppression of inlet velocity fluctuation by the secondary fuel corresponds to reduction of fine scale eddies and decreases Reynolds stress term caused by turbulent motion and entropy term due to energy dissipation rate.

\section{Acknowledgments}

This work is partially supported by Grant-in-Aid for Young Scientists (S) (No. 20676004) of Japan Society for the Promotion of Science and M. Shimura is supported by Grant-in-Aid for JSPS Fellows (No. 19 - 10540) of Japan Society for the Promotion of Science.

\section{References}

( 1 ) Rayleigh, L., The Theory of Sound, (1878 reedited in 1945), New York, Dover.

( 2 ) McManus, K. R., Poinsot, T. and Candel, S. M., A Review of Active Control of Combustion Instabilities, Progress in Energy and Combustion Science, Vol. 19 (1993), pp. 1-29.

( 3 ) Poinsot, T., Le Chatelier, C., Candel, S. M. and Esposito, E., Experimental Determination of the Reflection Coefficient of a Premixed Flame in a Duct, Journal of Sound and Vibration, Vol. 107, No. 2 (1986), pp. 265-278.

( 4 ) Harper, J., Johnson, C., Neumeier, Y., Lieuwen, T. and Zinn, B. T., Experimental Investigation of the Nonlinear Flame Response to Flow Disturbances in a Gas Turbine Combustor, AIAA-2001-0486.

( 5 ) Samaniego, J. M., Yip, B., Poinsot, T. and Candel, S, Low-Frequency Combustion Instability Mechanisms in a Side-Dump Combustor, Combustion and Flame, Vol. 94, No. 4 (1993), pp. 363-380.

( 6 ) Broda, J. C., Seo, S., Santro, R. J., Shirhattikar, G. and Yang, V., An Experimental Study of Combustion Dynamics of a Premixed Swirl Injector, Proceedings of the Combustion Institute, Vol. 27 (1998), pp. 1849-1856.

( 7 ) Balachandran, R., Ayoola, B. O., Kaminski, C. F., Dowling, A. P. and Mastorakos, E., Experimental Investigation of the Nonlinear Response of Turbulent Premixed Flames to Imposed Inlet Velocity Oscillations, Combustion and Flame, Vol. 143 (2005), pp. 37-55.

( 8 ) Li, Y., Tanahashi, M. and Miyauchi, T., Sound Generation in Chemically Reacting Mixing Layers, Transactions of the Japan Society of Mechanical Engineers, series B, Vol. 66, No. 648 (2000), pp. 2117-2124. 
( 9 ) Tanahashi, M., Tsukinari, S., Saitoh, T., Miyauchi, T., Choi, G.-M., Ikame, M., Kishi, T., Harumi, K. and Hiraoka, K., On the Sound Generation and its Controls in Turbulent Combustion Field, Proceedings of 3rd Symposium on Smart Control of Turbulence, (2002), pp. 149-160.

(10) Choi, G.-M., Li, Y., Tanahashi, M. and Miyauchi, T., Sound Generation Mechanism in Turbulent Mixing Layer, Turbulent Shear Flow Phenomena, Vol. 3, No. 2 (2003), pp. 729-734.

(11) Zhao, W. and Frankel, S. H., Numerical Simulations of Sound Radiated from an Axisymmetric Premixed Reacting Jet, Physics of Fluids, Vol. 13, No. 9 (2001), pp. 26712681.

(12) Tanahashi, M., Iwase, S. and Miyauchi, T., Appearance and Alignment with Strain Rate of Coherent Fine Scale Eddies in Turbulent Mixing Layer, Journal of Turbulence, Vol. 2, No. 6 (2001).

(13) Tanahashi, M., Kang, S. J., Miyamoto, T., Shiokawa, S. and Miyauchi, T., Scaling Law of Fine Scale Eddies in Turbulent Channel Flows up to $R e_{\tau}=800$, International Journal of Heat and Fluid Flow, Vol. 25 (2004), pp. 331-340.

(14) Dowling, A. P. and Morgans, A. S., Feedback Control of Combustion Oscillations, Annual Review of Fluid Mechanics, Vol. 37 (2005), pp. 151-182.

(15) Hong, B.-S., Ray, A. and Yang, V., Wide-Range Robust Control of Combustion Instability, Combustion and Flame, Vol. 128, No. 3 (2002), pp. 242-258.

(16) Lee, J. G., Kim, K. and Santavicca, D. A., Effect of Injection Location on the Effectiveness of an Active Control System Using Secondary Fuel Injection, Proceedings of the Combustion Institute, Vol. 28, No. 1 (2000), pp. 739-746.

(17) Tachibana, S., Zimmer, L., Kurosawa, Y. and Suzuki, K., Active Control of Combustion Oscillations in a Lean Premixed Combustor by Secondary Fuel Injection Coupling with Chemiluminescence Imaging Technique, Proceedings of the Combustion Institute, Vol. 31 (2007), pp. 3225-3233.

(18) Choi, G.-M., Tanahashi, M. and Miyauchi, T., Control of Oscillating Combustion and Noise Based on Local Flame Structure, Proceedings of the Combustion Institute, Vol. 30, No. 2 (2005), pp. 1807-1814.

(19) Tanahashi M., Murakami S., Miyauchi T. and Choi G.-M., Control of Oscillating Combustion and Measurements of Turbulent Flames, Proceedings of 5th Symposium on Smart Control of Turbulence, (2004), pp. 75-84.

(20) Uhm, J. H. and Acharya, S., Role of Low-Bandwidth Open-Loop Control of Combustion Instability Using a High-Momentum Air Jet-Mechanistic Details, Combustion and Flame, Vol. 147 (2006), pp. 22-31.

(21) Tanahashi, M., Inoue, S., Shimura, M., Taka, S., Choi, G.-M. and Miyauchi, T., Reconstructed 3D Flame Structures in Noise-Controlled Swirl-Stabilized Combustor, Experiments in Fluids, Vol. 45, No. 3 (2008), pp. 447-460.

(22) Dawson, J. R., Rodriguez-Martinez, V. M., Syred, N. and O'Doherty, T., The Effect of Combustion Instability on the Structure of Recirculation Zones in Confined Swirling Flames, Combustion Science and Technology, Vol. 177 (2005), pp. 2349-2371.

(23) Weigand, P., Meier, W., Duan, X. R., Stricker, W. and Aigner, M., Investigations of Swirl Flames in a Gas Turbine Model Combustor: I. Flow Field, Structures, Temperature and Species Distributions, Combustion and Flame, Vol. 144 (2006), pp. 205-224.

(24) Armitage, C. A., Balachandran, R., Mastorakos, E. and Cant, R. S., Investigation of the Nonlinear Response of Turbulent Premixed Flames to Imposed Inlet Velocity Oscillations, Combustion and Flame, Vol. 146 (2006), pp. 419-436.

(25) Prasad, A. K., Scheimpflug Stereocamera for Particle Image Velocimetry in Liquid Flows, Applied Optics, Vol. 34, No. 30 (1995), pp. 7092-7099.

(26) Prasad, A. K., Stereoscopic Particle Image Velocimetry,, Experiments in Fluids, Vol. 29 (2000), pp. 103-116. 
(27) Kumar, S. and Banerjee, S., Development and Application of a Hierarchical System for Digital Particle Image Velocimetry to Free-Surface Turbulence, Physics of Fluids, Vol. 10 (1998), pp. 160-177.

(28) Westerweel, J., Dabiri, D. and Garib, M., The Effect of a Discrete Window Offset on the Accuracy of Cross-Correlation Analysis of Digital PIV Recordings, Experiments in Fluids, Vol. 23 (1997), pp. 20-28.

(29) Tanahashi, M., Ootsu, M., Fukushima, M. and Miyauchi, T., Measurement of Coherent Fine Scale Eddies in Turbulent Mixing Layer by DPIV, Engineering Turbulence Modeling and Measurements, Vol. 5 (2002), pp. 525-534.

(30) Tanahashi, M., Hirayama, T., Taka, S. and Miyauchi, T., Measurement of Fine Scale Structure in Turbulence by Time-Resolved Dual-Plane Stereoscopic PIV, International Journal of Heat and Fluid Flow, Vol. 29, No. 3 (2008), pp. 792-802.

(31) Willert, C., Stereoscopic Digital Particle Image Velocimetry for Application in Wind Tunnel Flows, Measurement Science and Technology, Vol. 8 (1997), pp. 1465-1479.

(32) Peters, N., Turbulent Combustion, (2000) London, Cambridge University Press. 